30. Magister Gallus semble avoir enseigné l'astronomie et la médecine à l'université de Prague vers 1350-60; fut probablement le médecin de Charles IV, auquel il dédie un Vitae vivendae ratio, qui sera publiée à Prague en 1819 par Fr. Muller. A aussi écrit sur la peste, et sur les urines un traité qui aurait été imprimé au $15 \mathrm{e}$ siècle, mais n'est pas dans Klebs. - Neuburger II, 504.

31. Eustache Deschamps, env. 1346-1407, n'est pas médecin; bourgeois cossu, occupant de hautes charges à la cour de Charles V, il rime abondamment sur toutes gens et choses de son temps. Ami des larges «buveries» et des plats chargés de bons morceaux, - qui lui vaudront la goutte, - il y va néanmoins de son régime de santé: D'un notable enseignement pour continuer santé au corps d'omme, 226 vers débutant par: Pour vostre santé maintenir. Ce sont les habituelles règles de vie modérée, de la Lettre d'Aristote, du Régime de Salerne, d'Arnauld, sans rien de personnel. Publié en 1893, Oeuvres complètes, VIII, 339-346.

32. Maestro Gregorio (14e siècle), Fiori di medicina; p. p. Franc. Zambrini dans Scelte di curiosita letterarie. Bologne 1859. - Brinkmann, no. 28.

33. Heinricus de Wittenwil (vers 1430) du Toggenbourg, advocatus curiae, introduit dans son poème, Der Ring, un bref régime de santé; ses éléments se rattachant tantôt à Salerne, tantôt à la Lettre d'Aristote, tantôt à Avicenne. Publié 1931. - B. Milt, 290-293, avec bibliogr.

34. (Anonyme, époque pas indiquée). Ein altneapolitanisches Regimen Sanitatis; p. p. Adolf Mussafia, Sitzungsberichte der phil.-hist. Klasse der Kais. Akademie der Wissenschaften, Bd. 100, Mitteilungen aus romanischen Handschriften, Teil I; Vienne 1884. - Brinkmann, no. 18.

35. (Anonyme, début du 16e siècle) Regimen sanitatis (Vademecum of the famous Macheaths physicians); p. p. Cameron Gillies, Glasgow 1912. - Brinkmann, no. 9.

36. Bien que sa lère éd. soit de 1514, nous pouvons enfin accueillir dans notre liste la Rosa Gallica de Symphorien Champier (1471-1538); sur laquelle voir P. Allut, Symphorien Champier, 1859, p. 167-171. Ses sept livres couvrent le même domaine que les régimes de santé; $135 \mathrm{ff} .8^{\circ}: \ldots$ praecepta (empruntés à une dizaine d'auteurs nommés dans le titre, d'Hippocrate à Avicenne, et plusieurs autres) ... quae ad medicam artem rectamque vivendi formam plurimum conducunt ... - Wickersheimer $745 \mathrm{~s}$.

E. Olivier.

\title{
Eine Vorlesung über den Kaiserschnitt aus dem Anfang des I9. Jahrhunderts
}

Bekanntlich war der Kaiserschnitt in der vorantiseptischen Zeit eine gefährliche und gefürchtete Operation. Dennoch konnte er nicht immer umgangen werden, wenn es galt, bei besonderen Geburtsfällen, wo die Geburt auf den natürlichen Wegen unmöglich war, Mutter und Kind womöglich zu retten. 
Vor uns liegt eine Vorlesung über dieses Thema von dem damaligen Berner Chirurgen, Prof. R. A. Schiferli, der, wie es ja damals allgemein üblich war, neben der eigentlichen Chirurgie auch die Augenheilkunde und die Geburtshülfe und Gynäkologie zu seinem Fach zählte.

Der Ausdruck Kaiserschnitt ist in dieser Vorlesung nicht streng auf die Sectio caesarea im heutigen Sinn beschränkt; auch die Laparotomie bei Eileiterschwangerschaft wurde dazu gerechnet, obwohl gerade Schiferli diesen Eingriff lieber Gastrotomie nennen möchte.

Schiferli sagt: Den Kaiserschnitt wegen seiner Gefährlichkeit ganz zu verbannen, wäre Unsinn, weil eben sonst viele Mütter und Kinder einem unvermeidlichen Tode ausgesetzt wären.

Unter den Indikationen zu diesem Eingriff steht in erster Linie der Tod der Mutter am Ende der Schwangerschaft oder unter der Geburt bei lebensfähigem Kinde, eine Anzeige, deren Gebrauch sich im Dunkel des heroischen Zeitalters verliert.

Eine Reihe von berühmten Persönlichkeiten werden aufgeführt, die so zur Welt gekommen seien: Aesculap selbst, der Gott der Heilkunde, dann von historischen Personen: Scipio Africanus, Manlius, der Eroberer Karthagos, Julius Caesar, ein Graf von Linggow, später Abt von St. Gallen (man nannte ihn deswegen «ingenitus») Sancho major, König von Navarra, den ein Edler aus dem Leibe seiner von den Saracenen erschlagenen Mutter geschnitten habe, Gebhard, Graf von Bregenz, Doria der Feldherr, Plempius, der sich durch die Bekämpfung der Harveyschen Lehre vom Blutkreislauf auszeichnete und andere mehr.

Geistliche und weltliche Gesetzgeber und Herrscher haben zu allen Zeiten den Ärzten den Kaiserschnitt an der Toten ausdrücklich zur Pflicht gemacht; als Beispiel dient die Gesetzgebung des römischen Königs Numa Pompilius.

Für den Kaiserschnitt an der lebenden Frau gilt für Schiferli als Indikation: Beckenfehler, wenn der gerade Durchmesser des Beckeneinganges unter zwei Zoll beträgt (ca. $5 \mathrm{~cm}$ ).

Beispiele von Kaiserschnitt an der lebenden Frau finden sich erst vom Mittelalter an; auch diese sind unsicher; die erste sichere Mitteilung finde sich bei Fabricius Hildanus, der über einen von dem Chirurgen Trautmann 1610 mit glücklichem Erfolge ausgeführten Fall berichte. Erst 80 Jahre später sei die Operation wieder ausgeführt worden von Laubisch in Zittau, dann immer häufiger teils mit gutem, teils mit schlechtem Erfolge. 
Eine dritte Indikation sei die Schwangerschaft außerhalb der Gebärmutter, oder der Austritt der Frucht in die Bauchhöhle durch einen Riß in der Gebärmutter.

Um den Eingriff durchzuführen seien folgende Vorbereitungen notwendig: mehrere Bistouris, 2. Unterbindungsfaden, 3. Heftpflaster, 4. Charpie, 5. Schwämme, 6. Gerätschaften für die Bauchnaht, und 7. Binden.

Von Reinigungsmaßregeln finden wir keine Angaben.

Nach diesen Vorbereitungen wird mit einem convexen Bistouri oder Scalpell einen Zoll unter dem Nabel die Haut, das Zellgewebe, die weiße Linie (die Fascie) und das Bauchfell durchtrennt und unter Vermeidung des Urachus der Schnitt acht Zoll lang nach dem Schambein zu fortgesetzt und die blutenden Gefäße unterbunden. Lanverjeat rate zwar, einen Querschnitt zu machen; doch findet Schiferli, daß dadurch die Verletzung bedeutender und gefährlicher werde. (Bekanntlich hat der Querschnitt als angeblich neue Methode heute viele Anhänger.)

Darauf wird der Uterus ebenso eingeschnitten, der Zeigefinger der anderen Hand in die Wunde gebracht und auf diesem der Schnitt erweitert, so viel nötig ist. Es muß rasch operiert werden, um zu vermeiden, daß durch die Kontraktion des Uterus nach Abfluß des Fruchtwassers um den Fötus die Ausziehung erschwert werde. Sollte die Placenta in der Schnittgegend liegen, so tritt jetzt eine starke Blutung auf, die vorläufig nicht gestillt werden kann. Die Eingeweide, die sich etwa vordrängen, sind sorgfältig zurückzuhalten.

Der Uterus wurde also damals in Situ eröffnet und nicht vorgezogen.

Nun wird der Fötus an den Füßen gepackt und durch die Wunde extrahiert; die Nabelschnur unterbunden und durchschnitten. Die Extraktion muß rasch geschehen, damit die Blutung, besonders bei im Schnitt liegender Placenta, rasch gestillt werden kann.

Wenn jetzt kein Blut durch die Scheide abfließt muß man manuell ein allfälliges Hindernis, wie Eihautfetzen oder Blutgerinsel entfernen, um den Lochien den regelmäßigen Abfluß zu gestatten. Das Blut, das sich in die Bauchwunde ergossen hat, wird nach der Wunde und durch sie herausgestreichelt. Dann wäscht man die Bauchwunde ab und vereinigt sie genau durch eine Leibbinde. Nur wenn sich Därme vordrängen, muß die Bauchwunde genäht werden. (Von einer Naht der Uteruswunde wurde abgesehen.) Oft drängen sich nachträglich noch Darmschlingen in die Wunde und werden eingeklemmt, was einige Autoren als eine Hauptursache des häufigen üblen Ausganges ansehen. Jedenfalls soll man bei 
diesem Vorkommnis die Vereinigungsmittel entfernen, sobald sich Symptome der Einklemmung zeigen.

Auch bei glücklichem Ausgang bleibt die Gefahr des Bauchbruches, der man durch Tragen einer wohl passenden Leibbinde zuvorkommen muß. Die Kranke wird danach völlig wie eine Verwundete, und die Wunde wie eine einfache Schnittwunde behandelt.

Bei toter Schwangerer muß die Operation ganz unter den gleichen Kautelen verrichtet werden, weil sowohl die lebende Frucht geschont werden soll, als auch die Schwangere nur scheintot sein kann.

Bei Schwangerschaft außerhalb der Gebärmutter soll der Einschnitt besser etwas höher und zu der Seite, wo die Frucht liegt, zwischen der weißen Linie und der Spitze des Darmbeines gemacht werden; der Fötus komme dann entweder gleich, oder könne, nachdem der Sack, in dem er allenfalls enthalten sein könnte, aufgeschnitten worden sei, herausgenommen werden. Die Nachbehandlung ist bei der Gastrotomie dieselbe, wie beim Kaiserschnitt.

Nach diesen Angaben kann es sich bei den operierten Extrauterinschwangerschaften nur um schon weiter entwickelte Früchte gehandelt haben; diejenigen die sich in den ersten Wochen der Schwangerschaft durch plötzlich einsetzende foudroyante innere Blutung manifestieren, scheint man damals nicht als Schwangerschaften erkannt zu haben.

Der Kaiserschnitt wurde also damals im großen ganzen ähnlich wie heute ausgeführt; immerhin fehlt die relative Sicherheit, die durch die anti- und aseptische Operationsweise heute gewährleistet ist. Daß allerdings auch unter prekären äußeren Umständen der Ausgang ein glücklicher sein kann, zeigt ein Fall, der in einer älteren Zeitschrift veröffentlicht wurde und der aus der Mitte des vorigen Jahrhunderts stammt: Eine ungeduldige schwangere Frau befahl ihrem zwölfjährigen Töchterchen mit einem Küchenmesser ihr den Bauch aufzuschneiden und das Kind herauszunehmen. Das Mädchen gehorchte und nähte dann ebenfalls auf Weisung der Mutter, den Bauch mit gewöhnlichem Nähfaden wieder zu; die Mutter genas ohne weiteres.

Die bei Schiferli empfohlene Versorgung der Bauchwunde nur mit Heftpflaster entspricht der damals allgemein geübten und auch notwendigen offenen Wundbehandlung die noch Jahrzehnte später angewandt wurde. Wir sehen ja, daß eine allfällige Naht der Bauchwunde nur als Hülfsmaßregel bei stärkerem Vordrängen der Därme ins Auge gefaßt wurde. Ein Bericht des Bezirksarztes Winckel, des Vaters des späteren 
Münchner Gynäkologen Prof.v. Winckel, erzählt von einem Falle von fünfmaligem Kaiserschnitt, den der Berichterstatter an derselben Frau ausgeführt hat. Jedesmal wurde die Wunde offen behandelt; bei den späteren Operationen war der Uterus mit den Bauchdecken fest verwachsen, so daß er ohne Eröffnung der Bauchhöhle aufgeschnitten werden konnte.

Auch nach Einführung der infektionsbekämpfenden Methoden war der Kaiserschnitt immer noch gefährlich; sehr häufig kam es vor, daß bei einer späteren Schwangerschaft die durch eine einschichtige Naht vereinigte Uteruswunde riß, was zum Verblutungstode der Schwangeren führen konnte. Erst als nach dem Vorgange von Max Sänger in Prag diese einfache durch eine mehrschichtige Naht ersetzt wurde, kam diese Komplikation seltener vor. Auch hatte man gelernt, die Bauchhöhle durch Abstopfen mit Gazen vor dem Hereinlaufen von Fruchtwasser und Blut zu schützen.

Während zur Zeit Schiferlis die Vorderwand des Uterus, der in situ blieb, eröffnet wurde, pflegte man später, am Ende des letzten und Anfang des jetzigen Jahrhunderts den Schnitt in den dicken Fundusteil zu verlegen. Meist wurde ein Längsschnitt gemacht; Fritsch in Bonn empfahl einen queren Fundusschnitt, als weniger blutig; Polano öffnete den über die Schamfuge vorgeneigten Uterus an der Hinterwand, um spätere Darmverklebungen mit dem Schnitte zu vermeiden.

Seit etwa 25 Jahren wird die untere Uterushälfte, der Durchtrittsschlauch eingeschnitten. Dieser Schnitt hat den Vorteil, daß er nachher viel weniger den Zerrungen ausgesetzt ist, die beim Fundusschnitt infolge der Wochenbettscontraktionen und der Verkleinerung des Uterus bei der Involution vorhanden sein müssen. Zuerst hegte man hochgespannte Erwartungen, der suprasymphysäre Schnitt, wie man ihn nannte würde gestatten, nunmehr auch bei schon infiziertem Uterus mit weniger Gefahren einen Kaiserschnitt zu versuchen; auch wurden Methoden erfunden, die erlauben sollten, extraperitoneal vorzugehen; man probierte selbst von einem seitlichen Schnitte aus neben der Blase vorbeizukommen; aber alle diese Methoden konnten sich nicht halten da sie die Hoffnungen, die auf sie gesetzt wurden nicht oder doch nur sehr wenig erfüllten.

Neuerdings wird von verschiedenen Autoren lebhaft empfohlen, die Bauchdecken und den Uterus in seinem Durchtrittsschlauch quer zu eröffnen; man folgt dabei im ganzen dem Verlauf der Muskelfasern dieser 
Partie, und der Schnitt verläuft nicht quer zu den Blutgefäßen, sondern in ihrer Längsrichtung; die Verletzung ist nicht, wie Schiferli glaubte größer, sondern geringer als beim Längsschnitt, ebenso die Blutung.

Wie immer glaubt mancher heute auf einem definitiven Plan angekommen zu sein; wie immer wird wohl auch hier die Zukunft noch manches Neue bringen oder Altes wieder zu Ehren ziehen.

$$
\text { R. v. Fellenberg. }
$$

\section{Ein neuer Brief von Alfonso Corti (1822-1876)}

Als im Jahre 1876 Alfonso Corti in Corvino San Quirico bei Casteggio starb, hat die wissenschaftliche Welt von seinem Tod keine Notiz genommen; kein Nachruf ist uns erhalten, kein Bericht über seine so erfolgreich begonnenen wissenschaftlichen Studien ist überliefert worden. Der Name Corti, den wir uns heute aus der Lehre vom Bau des Spiralorganes im inneren $\mathrm{Ohr}$ gar nicht mehr wegdenken können, war damals anscheinend nur noch Wenigen bekannt. Etwas mehr als 20 Jahre, während derer Corti sich im wissenschaftlichen Schrifttum nicht hatte vernehmen lassen, genügten schon, um die Erinnerung an den Mann auszulöschen, dessen 1851 erschienenes Werk «Recherches sur l'organe de l'ouie des mammifères» für die Anatomie wie für die Physiologie gleich wichtig gewesen ist.

In mühevoller Arbeit haben Brückner, Schaffer und Pincherle lange nach dem Tode von Alfonso Corti versucht, dessen Lebenslauf aufzuklären und insbesondere die Zeiten und Orte seiner wissenschaftlichen Studien zu ermitteln. Ihre durchaus fragmentarisch gebliebenen Untersuchungen habe ich erst vor kurzem in wesentlichen Punkten ergänzen können auf Grund von Briefen Cortis, die sich im Nachlaß des Berner Histologen und Physiologen G. G. Valentin (1810-1883) fanden. ${ }^{1}$ Soweit Cortis Lebenslauf für die medizin-historische Forschung bedeutsam ist, liegt er nun einigermaßen klar vor uns; gleichwohl verdienen neue Quellen noch unsere Beachtung, dies besonders dann, wenn sie auch über die Persönlichkeit und die Wesensart von Alfonso Corti weiteren Aufschluß bringen.

1 E. Hintzsche, Alfonso Corti. Berner Beiträge zur Geschichte der Medizin und der Naturwissenschaften Nr. 3, 1944. 